\title{
Probability distribution analysis of M-QAM- modulated OFDM symbol and reconstruction of distorted data
}

\author{
Hyunseuk Yoo*, Frédéric Guilloud and Ramesh Pyndiah
}

\begin{abstract}
It is usually assumed that $N$ samples of the time domain orthogonal frequency division multiplexing (OFDM) symbols have an identical Gaussian probability distribution (PD) in the real and imaginary parts. In this article, we analyze the exact PD of M-QAM/OFDM symbols with $N$ subcarriers. We show the general expression of the characteristic function of the time domain samples of M-QAM/OFDM symbols. As an example, theoretical discrete PD for both QPSK and 16-QAM cases is derived. The discrete nature of these distributions is used to reconstruct the distorted OFDM symbols due to deliberate clipping or amplification close to saturation. Simulation results show that the data reconstruction process can effectively lower the error floor level.
\end{abstract}

Keywords: OFDM, discrete probability distribution, M-QAM, nonlinear amplifier, data reconstruction.

\section{Introduction}

A significant drawback of orthogonal frequency division multiplexing (OFDM)-based systems is their high peakto-average power ratio (PAPR) at the transmitter, requiring the use of a highly linear amplifier which leads to low power efficiency. For reasonable power efficiency, the OFDM signal power level should be close to the nonlinear area of the amplifier, going through nonlinear distortions and degrading the error performance.

The distortion can be introduced for two main reasons: nonlinear amplifier $[1,2]$ and/or deliberate clipping [3]. For the first case, if an OFDM symbol is amplified in the saturation area of an amplifier, its data recovery is not possible. For the second case, deliberate clipping makes an intentional noise which falls both in-band and out-of-band. In-band distortion results in an error performance degradation, while out-of-band radiation reduces spectral efficiency. Filtering methods can reduce out-of-band radiation, but also introduces peak regrowth of OFDM signals and increases the overall system impulse response $[4,5]$.

Several approaches have been investigated for mitigating the clipping noise with an amount of computational

\footnotetext{
* Correspondence: hyunseuk.yoo@telecom-bretagne.eu

Department of Signal and Communications, Telecom Bretagne, Technopole Brest Iroise - CS 83818, 29238 Brest cedex 3, France
}

complexity, such as iterative methods [6-10] and an oversampling method [11].

It is usually assumed that the time domain samples of OFDM symbols are complex Gaussian distributed, which is a very good approximation if the number of subcarriers is large enough. Furthermore, it is theoretically proved in $[12,13]$ that a bandlimited uncoded OFDM symbol converges weakly to a Gaussian random process as the number of subcarriers goes to infinity.

In this article, we derive the discrete Probability Distribution (PD) of the time domain samples of M-QAM/ OFDM symbols with a limited number of subcarriers. The discrete PD can be used to reconstruct distorted OFDM symbols. We focus on the in-band distortion which can be caused when OFDM symbols are amplified in the saturation area or when deliberate clipping is used to reduce the PAPR [3]. Note that the conventional Gaussian assumption cannot be used for the data recovery of distorted OFDM symbols. The article is organized as follows: In Section 2, we derive the PD of M-QAM modulated OFDM symbols. Using our derivation of PD, we consider the data reconstruction (DRC) method in the presence of a soft limiter in Section 3. Finally, we conclude this article in Section 4. 


\section{IDFT for M-QAM symbols}

An OFDM signal in the time domain is the sum of $N$ independent signals over sub-channels of equal bandwidth $1 /\left(T+T_{\mathrm{cp}}\right)$ and regularly spaced with frequency $1 /\left(T+T_{\mathrm{cp}}\right)$, where $T$ is the orthogonality period and $T_{\mathrm{cp}}$ is the duration of cyclic prefix.

At the transmitter, a frequency domain OFDM symbol $\mathbf{X}$ with $N$ samples $\mathbf{X}=\left\{X_{0}, X_{1}, \ldots, X_{N-1}\right\}$ is transformed via an $N$-point inverse discrete Fourier transform (IDFT) to a time domain OFDM symbol $\mathbf{x}$ with $N$ samples $\mathbf{x}=\left\{x_{0}, x_{1}, \ldots, x_{N-1}\right\}$ :

$$
x_{m}=\frac{1}{N} \sum_{l=0}^{N-1} X_{l} \cdot \exp \left(j \frac{2 \pi l m}{N}\right),
$$

where $m, l \in\{0,1, \ldots, N-1\}$. Note that the transmitted signal is made of the time domain OFDM symbol together with the cyclic prefix. Since the cyclic prefix is the copy of a part of $\mathbf{x}$, the derivation of the distribution of the samples in $\mathbf{x}$ completely determines the distribution of the transmitted signal.

We assume hereafter that all the frequency domain samples $X_{l}$ are uniformly distributed in the set of a square $M$-QAM constellation $\mathbf{S}$; for example: $\mathbf{S}=\left\{\frac{+1+j}{\sqrt{2}}, \frac{+1-j}{\sqrt{2}}, \frac{-1+j}{\sqrt{2}}, \frac{-1-j}{\sqrt{2}}\right\}$ in the QPSK case. In addition, the real and imaginary parts of $X_{l}$, denoted, respectively, $\hat{X}_{l} \triangleq \Re\left\{X_{l}\right\}, \breve{X}_{l} \triangleq \Im\left\{X_{l}\right\}$, are uniformly distributed as depicted in Figure 1. The minimum Euclidean distance of the constellation is given by $2 \tau$. Then, a general expression for the PD of $\left\{\hat{X}_{l}, \breve{X}_{l}\right\}, l \in\{0,1, \ldots, N-1\}$ is given by

$$
\operatorname{Pr}\left\{\hat{X}_{l}=(\sqrt{M}-2 k-1) \tau\right\}=\operatorname{Pr}\left\{\breve{X}_{l}=(\sqrt{M}-2 k-1) \tau\right\}=\frac{1}{\sqrt{M}}
$$

where $k \in\{0,1, \ldots, \sqrt{M}-1\}$.
The characteristic function of $\hat{X}_{l}$ and $\breve{X}_{l}, l \in\{0,1, \ldots$, $N-1\}$, is given by [14]

$$
\begin{aligned}
\varphi_{\hat{X}_{l}}(\omega) & =\varphi_{\breve{X}_{l}}(\omega) \\
& \triangleq \mathrm{E}\left[\exp \left(j \hat{X}_{l} \omega\right)\right] \\
& =\frac{1}{\sqrt{M}} \sum_{k=0}^{\sqrt{M}-1} \exp (j(\sqrt{M}-2 k-1) \tau \omega)
\end{aligned}
$$

where $E[\cdot]$ is the expectation operator. We will use this characteristic function in order to obtain the PD of time domain OFDM samples.

We first consider the real part $\hat{x}_{m} \triangleq \Re\left\{x_{m}\right\}$ given by

$$
\hat{x}_{m}=\frac{1}{N} \sum_{l=0}^{N-1}\left[\hat{X}_{l} \cdot \mathfrak{c}(l, m)+\breve{X}_{l} \cdot \mathfrak{s}(l, m)\right],
$$

where

$$
\mathfrak{c}(l, m) \triangleq \cos \left(\frac{-2 \pi l m}{N}\right)
$$

$\mathfrak{s}(l, m) \triangleq \sin \left(\frac{-2 \pi l m}{N}\right)$.

Given $l$ and $m$, since both $c(l, m)$ and $s(l, m)$ are constants, the characteristic functions of $\hat{X}_{l} \cdot \mathfrak{c}(l, m)$ and $\breve{X}_{l} \cdot \mathfrak{s}(l, m)$ are obtained as

$$
\begin{aligned}
& \varphi_{\widehat{X}_{\mathbb{I}_{i}} \mathfrak{c}(l, m)}(\omega)=\varphi_{\hat{X}_{l}}(\mathfrak{c}(l, m) \cdot \omega)=\frac{1}{\sqrt{M}} \sum_{k=0}^{\sqrt{M-1}} \exp (j(\sqrt{M}-2 k-1) \tau \cdot \mathfrak{c}(l, m) \cdot \omega), \\
& \varphi_{\breve{X}_{\mathfrak{l}} \mathfrak{s}(l, m)}(\omega)=\varphi_{\breve{X}_{l}}(\mathfrak{s}(l, m) \cdot \omega)=\frac{1}{\sqrt{M}} \sum_{k=0}^{\sqrt{M-1}} \exp (j(\sqrt{M}-2 k-1) \tau \cdot \mathfrak{s}(l, m) \cdot \omega) .
\end{aligned}
$$

Then, the characteristic function of $\hat{X}_{l} \cdot \mathfrak{c}(l, m)+\breve{X}_{l} \cdot \mathfrak{s}(l, m)$ is given by

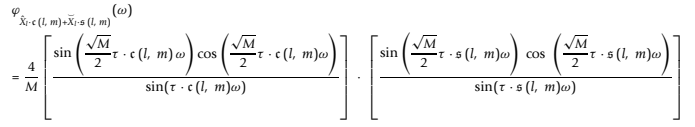

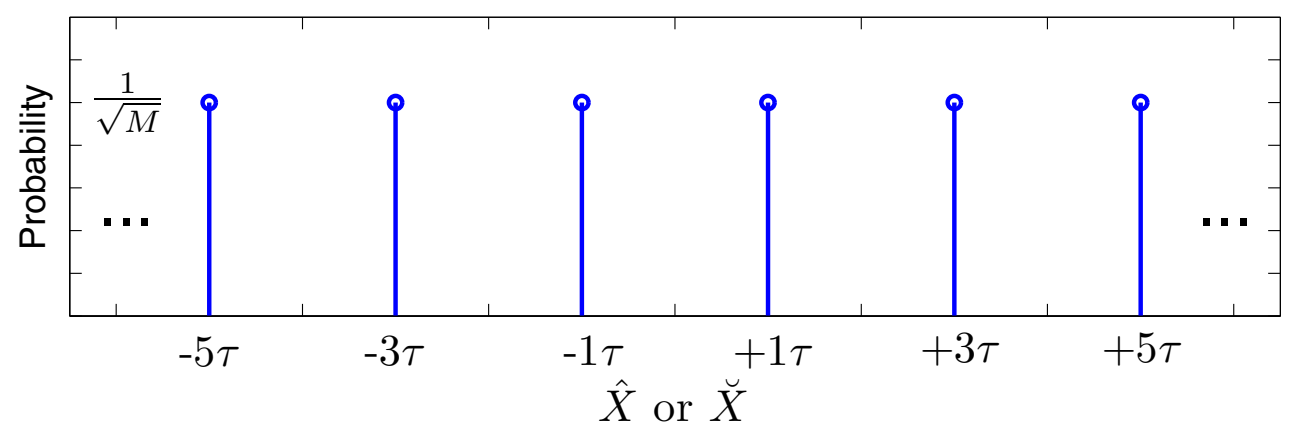

Figure 1 PD of the M-QAM symbol. PD of the M-QAM modulated symbol in each real or imaginary part, $\hat{X}$ or $\breve{X}$ 
which is proved in Appendix.

Since $\hat{X}_{l}$ and $\hat{X}_{l}, l \in\{0,1, \ldots, N-1\}$, are mutually independent, $\varphi_{N} \hat{x}_{m}(\omega)$ is given by Equation (7).

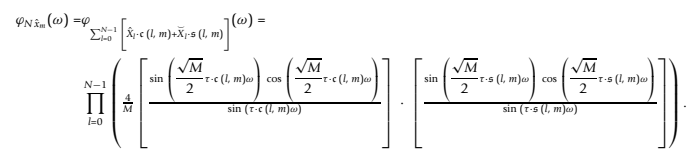

Therefore,

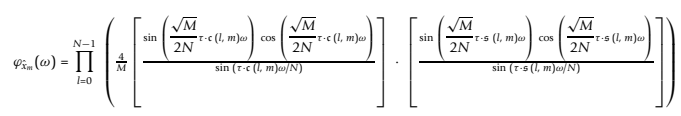

The general PD for M-QAM modulated OFDM symbols can be obtained by using inversion of characteristic function of (8), which is expressed as

$$
\operatorname{Pr}\left\{\hat{x}_{m}=x\right\}=\frac{1}{2 \pi} \int_{-\infty}^{\infty} \varphi_{\hat{x}_{m}}(\omega) \exp (-j \omega x) d \omega .
$$

Notice that, since $\varphi_{\hat{x}_{m}}(\omega)$ in (8) is a function of $m$, its PD is also a function of $m$. In other words, the mathematical expression of PD in (9) has a large number of different forms, depending on $m$. In the remainder of this article, to illustrate our reasoning, we restrict ourselves to the case where $m \in\left\{0, \frac{N}{4}, \frac{2 N}{4}, \frac{3 N}{4}\right\}$.

When $m \in\left\{0, \frac{N}{4}, \frac{2 N}{4}, \frac{3 N}{4}\right\}$, Equation (8) is reduced to

$$
\begin{aligned}
\varphi_{\hat{x}_{m}}(\omega) & =\left(\frac{2 \sin \left(\frac{\sqrt{M}}{2 N} \tau \omega\right) \cos \left(\frac{\sqrt{M}}{2 N} \tau \omega\right)}{\sqrt{M} \sin (\tau \omega / N)}\right)^{N} \\
& =\left(\frac{\sin (\sqrt{M} \tau \omega / N)}{\sqrt{M} \sin (\tau \omega / N)}\right)^{N} .
\end{aligned}
$$

As a function of $M$, Equation (10) represents the characteristic function of $\hat{x}_{m} \triangleq \Re\left\{x_{m}\right\}$. We proceed further the PD derivation for two representative examples of modulation scheme: QPSK $(M=4)$ and 16-QAM $(M=16)$.

\subsection{QPSK case}

In the QPSK case $(M=4)$, Equation (10) turns into

$$
\begin{aligned}
\varphi_{\hat{x}_{m}}(\omega)= & {[\cos (\tau \omega / N)]^{N} } \\
= & \frac{1}{2^{N}}\left(\begin{array}{c}
N \\
N / 2
\end{array}\right)+\frac{2}{2^{N}} \sum_{k=0}^{\frac{N}{2}-1}\left(\begin{array}{l}
N \\
k
\end{array}\right) \cos \left(\frac{(N-2 k) \tau \omega}{N}\right), \\
= & \frac{1}{2^{N}}\left(\begin{array}{c}
N \\
N / 2
\end{array}\right)+\frac{1}{2^{N}} \sum_{k=0}^{\frac{N}{2}-1}\left(\begin{array}{c}
N \\
k
\end{array}\right) \\
& \cdot\left[\exp \left(\frac{j(N-2 k) \tau \omega}{N}\right)+\exp \left(\frac{-j(N-2 k) \tau \omega}{N}\right)\right] .
\end{aligned}
$$

Referring to Equations (2) and (3), the discrete PD of $\operatorname{Pr}\left\{\hat{x}_{m}\right\}, \operatorname{Pr}\left\{\hat{x}_{m}\right\}$, is given by

$$
\begin{aligned}
& \operatorname{Pr}\left\{\hat{x}_{m}=0\right\}=\frac{1}{2^{N}}\left(\begin{array}{c}
N \\
N / 2
\end{array}\right), \\
& \operatorname{Pr}\left\{\hat{x}_{m}=\tau\left(1-\frac{2 k}{N}\right)\right\}=\operatorname{Pr}\left\{\hat{x}_{m}=\tau\left(\frac{2 k}{N}-1\right)\right\}=\frac{1}{2^{N}}\left(\begin{array}{l}
N \\
k
\end{array}\right),
\end{aligned}
$$

where $k \in\left\{0,1, \ldots, \frac{N}{2}-1\right\}$.

Similarly, the PD of $\breve{x}_{m} \triangleq \Im\left\{x_{m}\right\}$ can be derived as $\operatorname{Pr}\left\{\breve{x}_{m}\right\}=\operatorname{Pr}\left\{\hat{x}_{m}\right\}$.

\subsection{6-QAM case}

In the 16-QAM case $(M=16), \varphi_{\hat{x}_{m}}(\omega)$ from $(10)$ is given by

$$
\begin{aligned}
\varphi_{\hat{x}_{m}}(\omega) & =\left[\cos \left(\frac{2 \tau \omega}{N}\right)\right]^{N} \cdot\left[\cos \left(\frac{\tau \omega}{N}\right)\right]^{N}=\left[2\left(\cos \left(\frac{\tau \omega}{N}\right)\right)^{3}-\cos \left(\frac{\tau \omega}{N}\right)\right]^{N} \\
& =\sum_{k=0}^{N}\left(\begin{array}{l}
N \\
k
\end{array}\right)(-1)^{k} \cdot 2^{N-k} \cdot\left(\cos \left(\frac{\tau \omega}{N}\right)\right)^{3 N-2 k},
\end{aligned}
$$

where

$$
\begin{aligned}
\left(\cos \left(\frac{\tau \omega}{N}\right)\right)^{3 N-2 k}= & \frac{1}{2^{3 N-2 k}}\left(\begin{array}{c}
3 N-2 k \\
\frac{3 N-2 k}{2}
\end{array}\right)+\frac{1}{2^{3 N-2 k}} \sum_{t=0}^{\frac{3 N-2 k}{2}-1}\left(\begin{array}{c}
3 N-2 k \\
t
\end{array}\right) \\
& \cdot\left[\exp \left(\frac{j \tau \omega(3 N-2 k-2 t)}{N}\right)+\exp \left(\frac{-j \omega(3 N-2 k-2 t)}{N}\right)\right] .
\end{aligned}
$$

Using (14), Equation (13) is expressed as follows:

$$
\begin{aligned}
\varphi_{\hat{x}_{m}}(\omega)= & \sum_{k=0}^{N}\left(\begin{array}{l}
N \\
k
\end{array}\right) \cdot\left(\begin{array}{c}
3 N-2 k \\
\frac{3 N-2 k}{2}
\end{array}\right) \cdot(-1)^{k} \cdot \frac{2^{N-k}}{2^{3 N-2 k}} \\
& +\sum_{k=0}^{N} \sum_{t=0}^{\frac{3 N-2 k}{2}-1}\left(\begin{array}{c}
N \\
k
\end{array}\right) \cdot\left(\begin{array}{c}
3 N-2 k \\
t
\end{array}\right) \cdot(-1)^{k} \cdot \frac{2^{N-k}}{2^{3 N-2 k}} \\
& \cdot\left[\exp \left(\frac{j \tau \omega(3 N-2 k-2 t)}{N}\right)+\exp \left(\frac{-j \tau \omega(3 N-2 k-2 t)}{N}\right)\right] .
\end{aligned}
$$

The first term in Equation (15) gives the PD of $\hat{x}_{m}$ :

$$
\operatorname{Pr}\left\{\hat{x}_{m}=0\right\}=\sum_{k=0}^{N}\left(\begin{array}{l}
N \\
k
\end{array}\right) \cdot\left(\begin{array}{c}
3 N-2 k \\
\frac{3 N-2 k}{2}
\end{array}\right) \cdot(-1)^{k} \cdot \frac{2^{N-k}}{2^{3 N-2 k}} .
$$

For the second term in Equation (15), let $p=k+t$, then

$$
\begin{gathered}
\operatorname{Pr}\left\{\hat{x}_{m}=\frac{\tau(3 N-2 p)}{N}\right\}=\operatorname{Pr}\left\{\hat{x}_{m}=\frac{-\tau(3 N-2 p)}{N}\right\} \\
=\sum_{k=0}^{\min (N, p)}\left(\begin{array}{c}
N \\
k
\end{array}\right) \cdot\left(\begin{array}{c}
3 N-2 k \\
p-k
\end{array}\right) \cdot(-1)^{k} \cdot \frac{2^{N-k}}{2^{3 N-2 k}}
\end{gathered}
$$

where $p \in\left\{0,1, \ldots, \frac{3 N}{2}-1\right\}$.

Similarly, we can obtain $\operatorname{Pr}\left\{\breve{x}_{m}\right\}=\operatorname{Pr}\left\{\hat{x}_{m}\right\}$.

\subsection{Graphical comparison}

Figures 2 and 3 represent the comparison between the estimated (upper) and theoretical (lower) PDs of $m \in\left\{0, \frac{N}{4}, \frac{2 N}{4}, \frac{3 N}{4}\right\}$ for the QPSK and the 16-QAM case, 

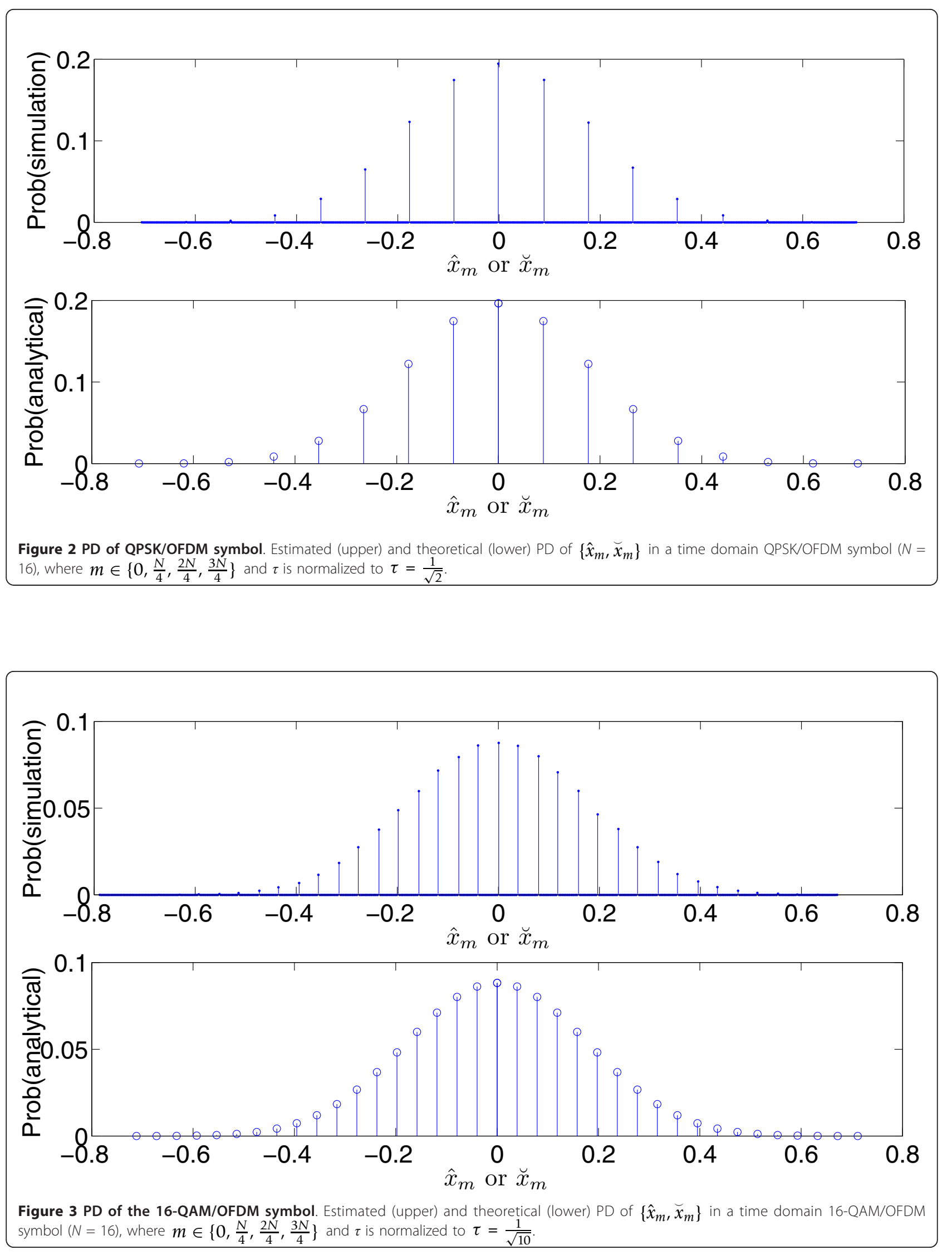
respectively, where $m \in\left\{0, \frac{N}{4}, \frac{2 N}{4}, \frac{3 N}{4}\right\}$. The estimated PD matches the theoretical PD.

Note that these results describe the discrete distribution of $\left\{\hat{x}_{m}, \breve{x}_{m}\right\}$, which is not continuous Gaussian distribution. In the following section, we will use the discrete nature of the distribution to reconstruct distorted OFDM symbols.

\section{Application to DRC}

In this section, we show that PD analysis can be applicable to DRC at the receiver. We consider a deliberately clipped OFDM symbol [3] or an OFDM symbol which operates in the saturation area of an amplifier. Note that these kinds of distorted OFDM symbols yield an error floor, depending on the saturation level.

\subsection{Soft clipping}

In order to illustrate the DRC concept, we consider hereafter an example of a QPSK case without loss of generality. Figure 4 represents the constellation of $X_{l}$ (frequency domain), where $l \in\{0,1, \ldots, N-1\}$. Using Equation (12), the constellation of $x_{m}$ (time domain), $m \in\left\{0, \frac{N}{4}, \frac{2 N}{4}, \frac{3 N}{4}\right\}$, is depicted in Figure 5. We assume that a soft limiter simply clips the OFDM symbol $x_{m}$ as follows [3]:

$$
\bar{x}_{m}=\left\{\begin{aligned}
x_{m \prime}, & \text { for }\left|x_{m}\right| \leq \bar{A} \\
\bar{A} \cdot \frac{x_{m}}{\left|x_{m}\right|}, & \text { for }\left|x_{m}\right|>\bar{A},
\end{aligned}\right.
$$

where $\bar{A}$ is the maximum permissible amplitude limit, and $m \in\{0,1, \ldots, N-1\}$. Note that $\bar{A}$ can be seen as the saturated amplitude of the amplifier.

As the soft limiter is processed on $x_{m}$, the clipping boundary can be observed on the constellation of $x_{m}$ as depicted in Figure 6 for $m \in\left\{0, \frac{N}{4}, \frac{2 N}{4}, \frac{3 N}{4}\right\}$. In this figure, the circle represents the maximum permissible amplitude $(\bar{A}=0.24)$ as a clipping threshold. Therefore, the external constellation points (outside the circle) are projected on the circle due to the clipping process. As a

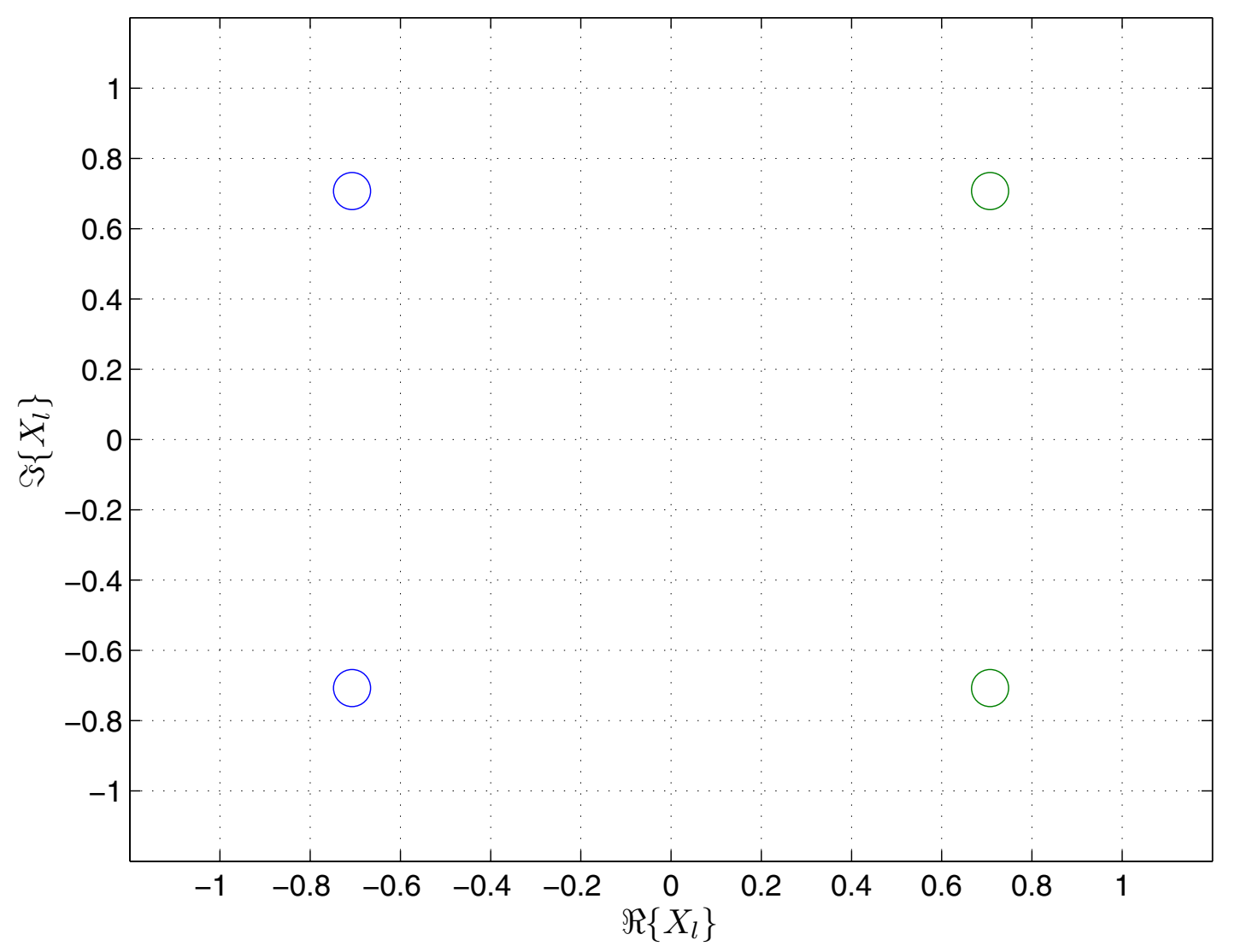

Figure 4 Constellation of $X_{I}$ (QPSK modulation). Constellation of $X_{I}$ (QPSK modulation), where $/ \in\{0,1, \ldots, N-1\}$. 


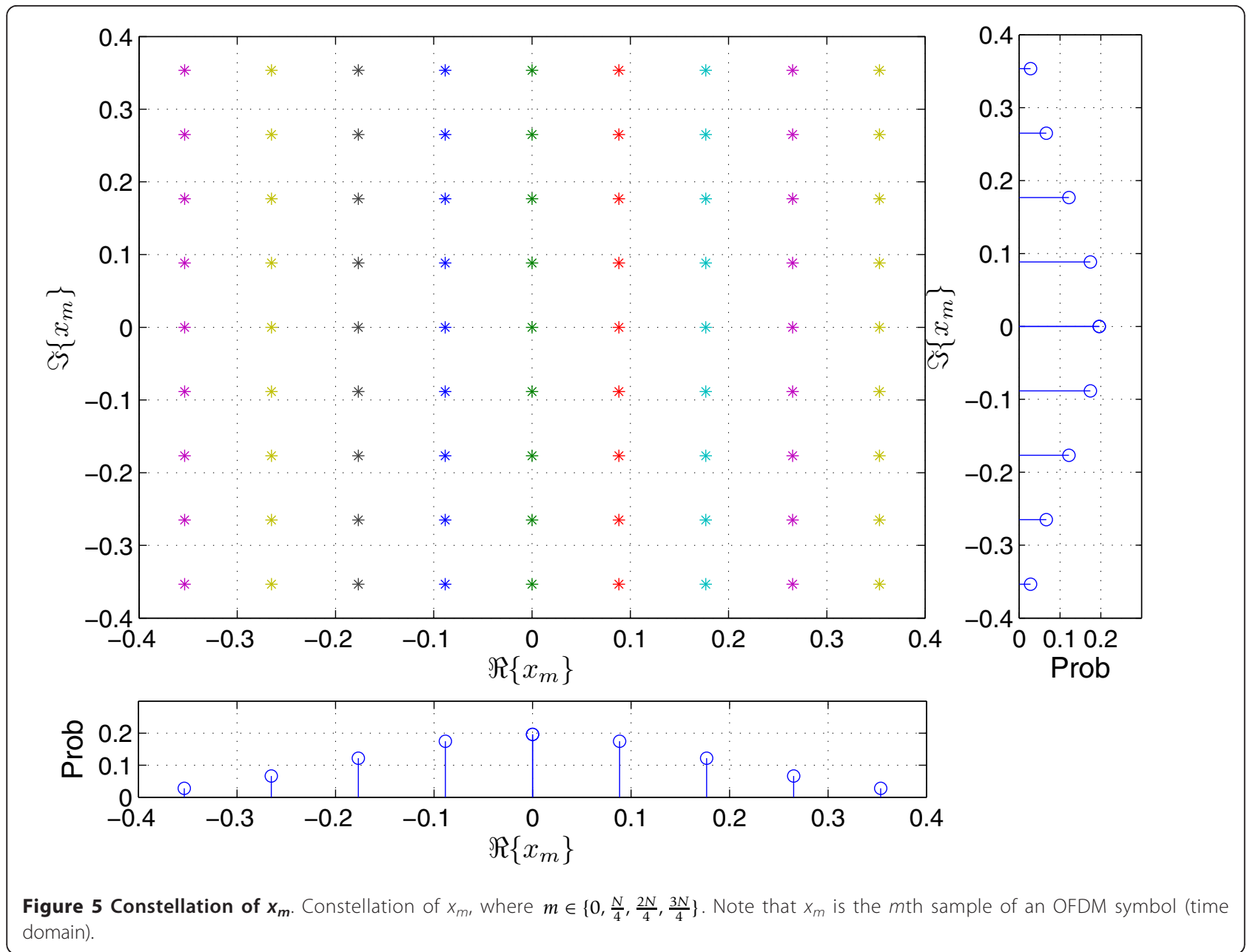

simple example, the constellation points " $\Delta$ " are projected on the circle and the points " $\square$ " are transmitted instead of " $\Delta$ ".

\subsection{Data ReConstruction}

Let $\mathbf{s}$ denotes the constellation of $\bar{x}_{m}$ (see " $\diamond$ " and " $\square$ " in Figure 6), where $m \in\left\{0, \frac{N}{4}, \frac{2 N}{4}, \frac{3 N}{4}\right\}$. In this example, the number of " $\nabla$ " is $n_{d}=21$ and the number of " $\square$ " is $n_{s}=24$. Therefore, the length of the vector $\mathbf{s}$ is $K=n_{d}$ $+n_{s}=21+24=45$ such as $\mathbf{s}=\left\{s_{1}, s_{2}, \ldots, s_{45}\right\}$. The set $\mathbf{s}$ is divided into two subsets: $\mathbf{s}_{d}$ and $\mathbf{s}_{s}$

$$
\mathbf{s}=\{\underbrace{s_{1}, s_{2}, \ldots, s_{n_{d}}}_{\mathbf{s}_{d}}, \underbrace{s_{n_{d}+1}, s_{n_{d}+2, \ldots, s_{K}}}_{\mathbf{s}_{s}}\},
$$

where $\mathbf{s}_{d}$ is the constellation inside the circle (" $\diamond$ " in Figure 6) and $\mathbf{s}_{s}$ is the constellation on the circle (" $\square$ " in Figure 6).

We consider two kinds of channel: noiseless and AWGN channels. Over a noiseless channel, if a received sample $r_{m}=\bar{x}_{m} \in \mathbf{s}_{d}, r_{m}$ indicates one of " $\diamond$ " marks.
Then, DRC is not performed, since $\bar{x}_{m}=x_{m}$. If a received sample $r_{m}=\bar{x}_{m} \in \mathbf{s}_{s}, r_{m}$ indicates one of " $\square$ " marks. Then DRC is performed by expanding this “ $\square$ " mark to the expected position " $\Delta$ " through the line as illustrated in Figure 7.

Over an AWGN channel, we can use maximum likelihood detection to reconstruct data. A priori probability $\operatorname{Pr}\left\{\bar{x}_{m}=s_{k}\right\}, k \in\{1,2, \ldots, K\}$ can be obtained from the joint probabilities of $\hat{x}_{m}$ and $\breve{x}_{m}, m \in\left\{0, \frac{N}{4}, \frac{2 N}{4}, \frac{3 N}{4}\right\}$, by using Equation (12). Through the AWGN channel, a noisy sample $r_{m}=\bar{x}_{m}+w_{m}$ is received, where $w_{m}$ is a complex Gaussian random variable with the AWGN standard deviation $\sigma$. Using a maximum likelihood criterion, the most probable constellation symbol $\Phi_{m} \in \mathbf{s}$ is obtained as follows:

$$
\begin{aligned}
\phi_{m} & =\arg \max _{s_{k} \in \mathbf{s}} \operatorname{Pr}\left\{\bar{x}_{m}=s_{k}\right\} \cdot \operatorname{Pr}\left\{r_{m} \mid \bar{x}_{m}=s_{k}\right\} \\
& =\arg \max _{s_{k} \in \mathbf{s}} \frac{\operatorname{Pr}\left\{\bar{x}_{m}=s_{k}\right\}}{\sigma \sqrt{\pi}} \exp \left(-\frac{\left|r_{m}-s_{k}\right|^{2}}{\sigma^{2}}\right) .
\end{aligned}
$$




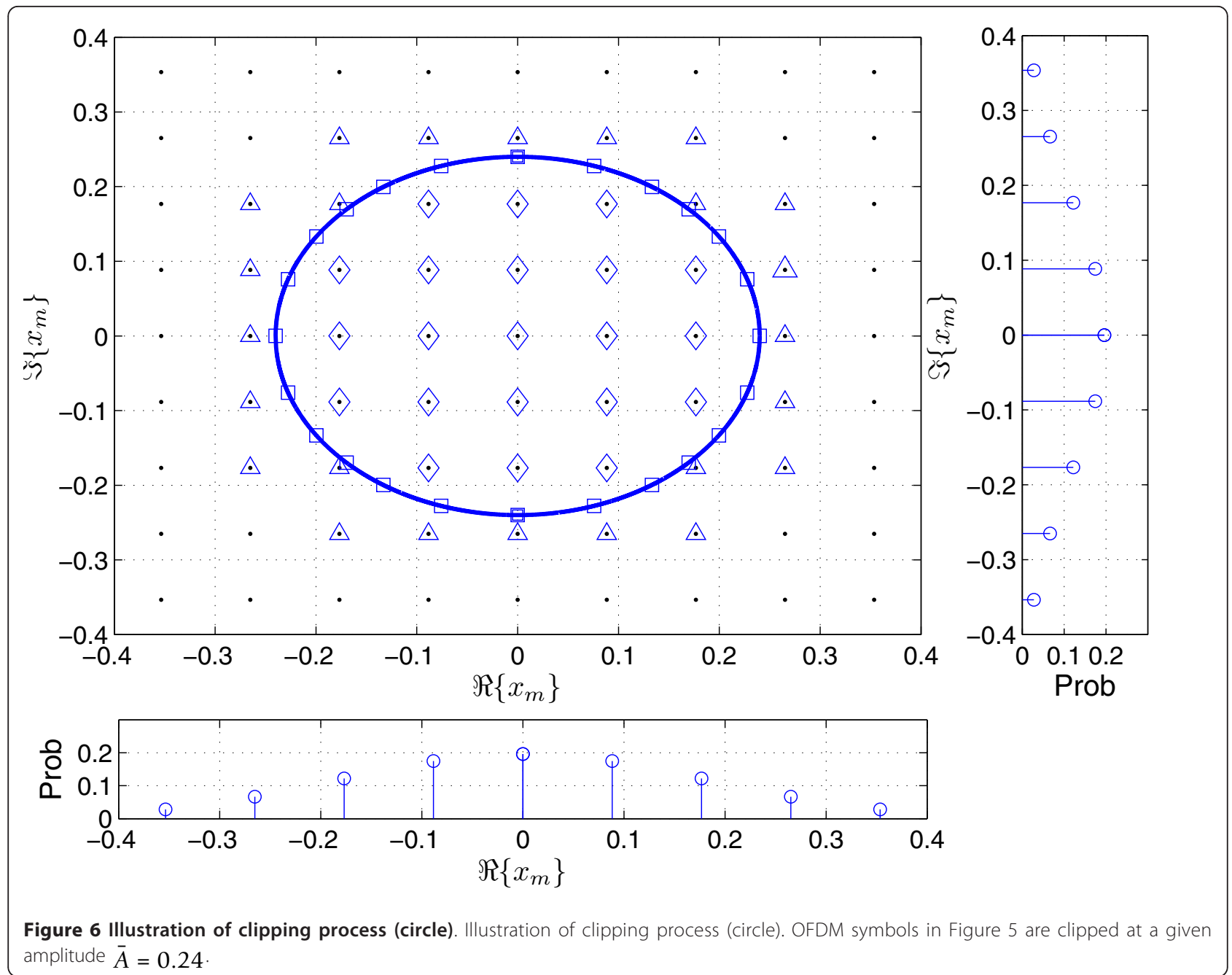

DRC is processed as follows: If $\varphi_{m}$ is positioned inside the circle $\left(\varphi_{m} \in \mathbf{s}_{d}\right), r_{m}$ is not modified. If $\varphi_{m}$ is positioned on the circle, it means that $\varphi_{m}$ corresponds to a $\square$ mark; then its corresponding $\Delta$ mark is the reconstructed value of $r_{m}$.

\subsection{Numerical results}

Figure 8 shows the influence of DRC on the QPSK symbol error rate (SER). For the simulation, QPSK/OFDM symbols are considered with $N=16$. A soft limiter clips the OFDM symbol at $\bar{A}=\{0.22,0.23,0.24,0.25\}$. In this figure, the dashed lines represent the original OFDM system (clipping without DRC) and the solid lines represent the DRC case.

The figure shows that DRC can effectively lower the error floor in the presence of a soft limiter or a saturated nonlinear amplifier, when $N$ is small. Note that the performance improvements depend on the clipping threshold $\bar{A}$, since the constellation of $\left\{x_{0}, x_{N / 4}, x_{2}\right.$ N/4, $\left.x_{3 N / 4}\right\}$ is fixed.
Regardless of the number of subcarriers $N$, the PD analysis is always valid, and is given by Equations (12), (16), and (17). However, since only four subcarriers are used for DRC, the application for large $N$ will be less effective. Nevertheless, for higher values of $N$, it may be worth calculating Equation (9) for some more values of $m$.

\section{Conclusion}

We analyze the PD of M-QAM-modulated OFDM symbols. Theoretically, the PD of the $m$ th OFDM symbol with $N$ subcarriers is not continuous Gaussian, and the PD is a function of $m$, where $m \in\{0,1 \ldots, N-1\}$. We provide a general form of the PD for $m \in\{0,1 \ldots, N$ $1\}$, and also derive the PD for exemplary cases of $m \in\left\{0, \frac{N}{4}, \frac{2 N}{4}, \frac{3 N}{4}\right\}$. The discrete nature of the distribution can be used to reconstruct the distorted OFDM symbols in the presence of a soft limiter or a saturated nonlinear amplifier, by using the maximum likelihood criterion. The reconstruction of OFDM symbols lowers the error floor level. 


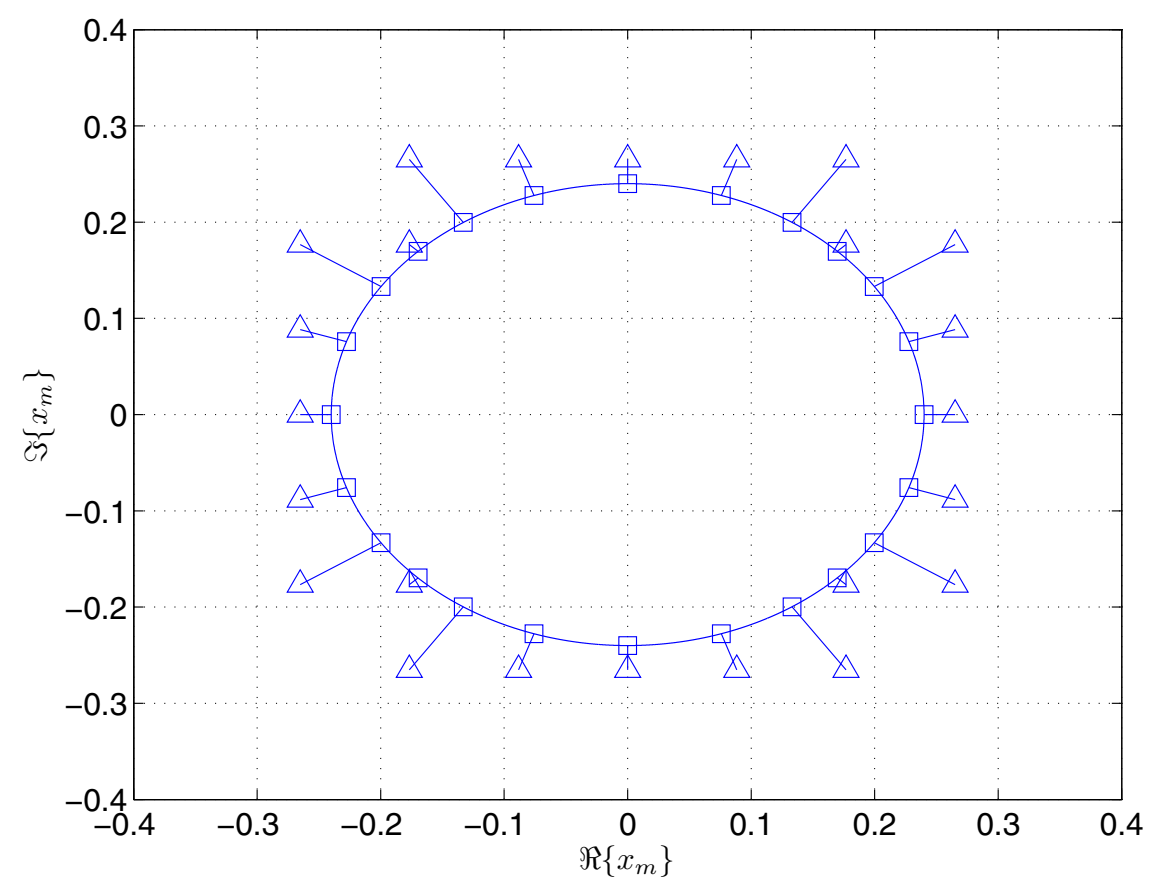

Figure 7 DRC. DRC from the clipped OFDM symbols " $\square$ " to the original constellations " $\triangle$ ".

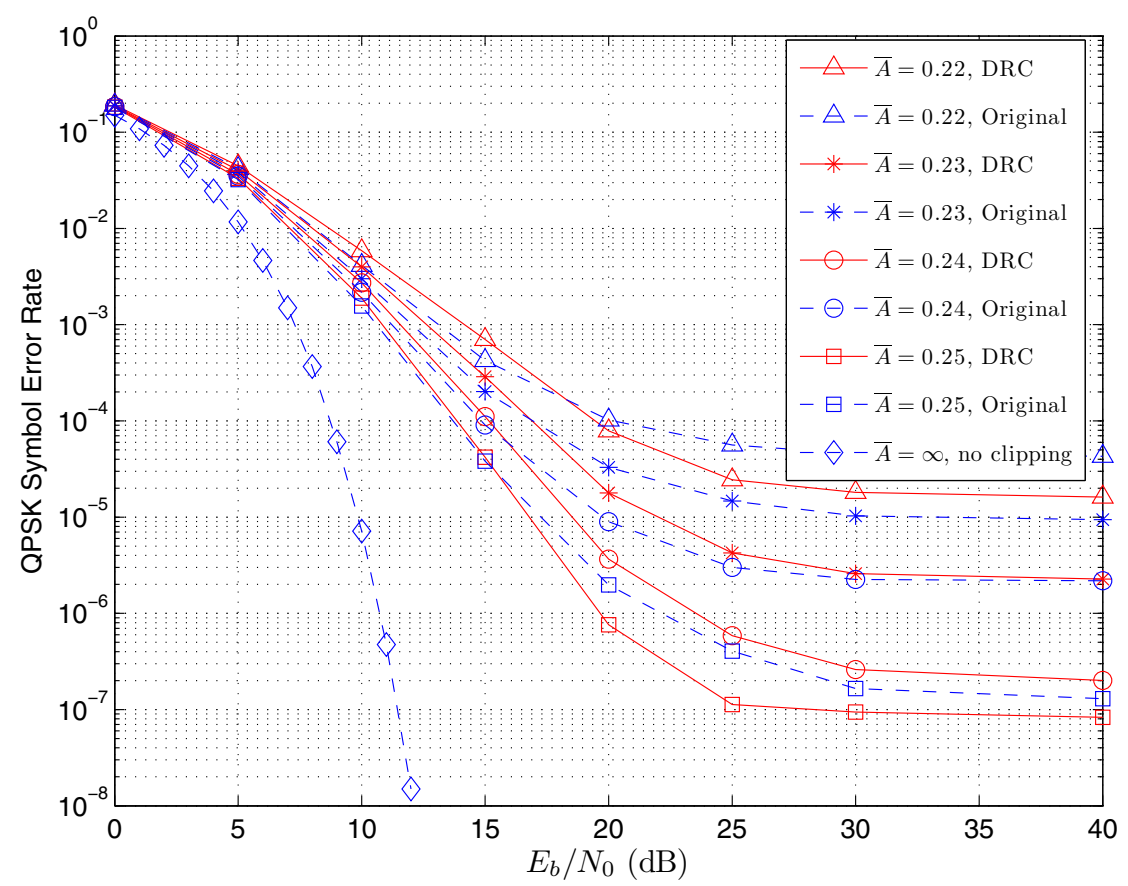

Figure 8 QPSK SER with and without DRC. QPSK SER with and without DRC, where QPSK modulated OFDM symbols $(N=16)$ are considered. A soft limiter clips the OFDM symbol at $\bar{A}=\{0.22,0.23,0.24,0.25, \infty\}$. Note that the case of $\bar{A}=\infty$ represents that OFDM symbols are not clipped. 


\section{Appendix}

Let $C_{1} \triangleq \tau \cdot \mathfrak{c}(l, m) \cdot \omega$ and $C_{2} \triangleq \tau \cdot \mathfrak{s}(l, m) \cdot \omega$. Then, Equation (6) is expressed as

$$
\begin{aligned}
& \varphi_{\overleftarrow{X}_{l} \in(l, m)+\breve{X}_{i}: \mathfrak{s}(l, m)}(\omega) \\
= & \frac{1}{M}\left[\sum_{k=0}^{\sqrt{M}-1} \exp \left(j(\sqrt{M}-2 k-1) C_{1}\right)\right] \cdot\left[\sum_{k=0}^{\sqrt{M}-1} \exp \left(j(\sqrt{M}-2 k-1) C_{2}\right)\right] .
\end{aligned}
$$

The first term in (21) is given by

$$
\begin{aligned}
& \sum_{k=0}^{\sqrt{M}-1} \exp \left(j(\sqrt{M}-2 k-1) C_{1}\right) \\
= & \sum_{k=0}^{\frac{\sqrt{M}}{2}-1} \exp \left(j(\sqrt{M}-2 k-1) C_{1}\right)+\sum_{\frac{\sqrt{M}}{2}}^{\sqrt{M}-1} \exp \left(j(\sqrt{M}-2 k-1) C_{1}\right) \\
= & \sum_{k=0}^{\frac{\sqrt{M}}{2}-1}\left[\cos \left((\sqrt{M}-2 k-1) C_{1}\right)+j \sin \left((\sqrt{M}-2 k-1) C_{1}\right)\right] \\
& +\sum_{k=0}^{\frac{\sqrt{M}}{2}-1}\left[\cos \left((\sqrt{M}-2 k-1) C_{1}\right)+j \sin \left((\sqrt{M}-2 k-1) C_{1}\right)\right] \\
= & 2 \cdot \sum_{k=0}^{\frac{\sqrt{M}}{2}-1}\left[\cos \left((\sqrt{M}-2 k-1) C_{1}\right)\right] .
\end{aligned}
$$

In a similar way, the second term in (21) is given by

$$
\sum_{k=0}^{\sqrt{M}-1} \exp \left(j(\sqrt{M}-2 k-1) C_{2}\right)=2 \cdot \sum_{k=0}^{\frac{\sqrt{M}}{2}-1}\left[\cos \left((\sqrt{M}-2 k-1) C_{2}\right)\right] .
$$

Then, using (22) and (23), Equation (21) is rewritten as

$$
\begin{aligned}
& \varphi_{\hat{X}_{r} \cdot c(l, m)+\breve{X}_{l} \cdot \mathfrak{s}(l, m)}(\omega) \\
= & \frac{4}{M}\left(\sum_{k=0}^{\frac{\sqrt{M}}{2}-1}\left[\cos \left((\sqrt{M}-2 k-1) C_{1}\right)\right]\right) \cdot\left(\sum_{k=0}^{\frac{\sqrt{M}}{2}-1}\left[\cos \left((\sqrt{M}-2 k-1) C_{2}\right)\right]\right) \\
= & \left.\frac{4}{M}\left(\sum_{k=0}^{\frac{\sqrt{M}}{2}-1}\left[\cos ((2 k+1)) C_{1}\right)\right]\right) \cdot\left(\sum_{k=0}^{\frac{\sqrt{M}}{2}-1}\left[\cos \left((2 k+1) C_{2}\right)\right]\right) .
\end{aligned}
$$

Using an arithmetic formula [15] denoting a finite sum of cosines given by

$$
\sum_{k=0}^{n} \cos (k a+b)=\frac{\sin \left(\frac{n+1}{2} a\right) \cos \left(\frac{a n}{2}+b\right)}{\sin \frac{a}{2}}, \text { where } n \in\{1,2, \ldots\}
$$

Equation (24) is written as

$$
\begin{aligned}
& \varphi_{\hat{X}_{l} \cdot \mathfrak{c}(l, m)+\breve{X}_{l} \cdot \mathfrak{s}(l, m)}(\omega) \\
= & \frac{4}{M}\left[\frac{\sin \left(\frac{\sqrt{M}}{2} C_{1}\right) \cos \left(\frac{\sqrt{M}}{2} C_{1}\right)}{\sin \left(C_{1}\right)}\right] \cdot\left[\frac{\sin \left(\frac{\sqrt{M}}{2} C_{2}\right) \cos \left(\frac{\sqrt{M}}{2} C_{2}\right)}{\sin \left(C_{2}\right)}\right] .
\end{aligned}
$$

\section{Competing interests}

The authors declare that they have no competing interests.

Received: 10 March 2011 Accepted: 19 December 2011 Published: 19 December 2011

\section{References}

1. HG Ryu, JS Park, JS Park, Threshold IBO of HPA in the predistorted OFDM communication system. IEEE Trans Broadcast. 50(4), 425-428 (2004). doi:10.1109/TBC.2004.837878

2. N Chen, GT Zhou, H Qian, Power efficiency improvements through peak-toaverage power ratio reduction and power amplifier linearization. EURASIP J Adv Signal Process. 2007, Article ID 20463, 7 (2007)

3. H Ochiai, H Imai, Performance of the deliberate clipping with adaptive symbol selection for strictly band-limited OFDM systems. IEEE J Sel Areas Commun. 18, 2270-2277 (2000). doi:10.1109/49.895032

4. X Li, LJ Cimini, Effect of clipping and filtering on the performance of OFDM. IEEE Commun Lett. 2, 131-133 (1998). doi:10.1109/4234.673657

5. SH Han, JH Lee, An overview of peak-to-average power ratio reduction techniques for multicarrier transmission. IEEE Wire Commun. 12, 56-65 (2005). doi:10.1109/MWC.2005.1421929

6. D Kim, GL Stuber, Clipping noise mitigation for OFDM by decision aided reconstruction. IEEE Commun Lett. 3, 4-6 (1999). doi:10.1109/4234.740112

7. H Chen, AM Haimovich, Iterative estimation and cancellation of clipping noise for OFDM signals. IEEE Commun Lett. 7(7), 305-307 (2003). doi:10.1109/LCOMM.2003.814720

8. J Tong, L Ping, Z Zhang, B VK, Iterative Soft compensation for OFDM systems with clipping and superposition coded modulation. IEEE Commun Trans. 58(10), 2861-2870 (2010)

9. J Armstrong, Peak-to-average power reduction for OFDM by repeated clipping and frequency domain filtering. Electron Lett. 38, 246-247 (2002). doi:10.1049/el:20020175

10. W Rave, P Zillmann, G Fettweis, Iterative correction and decoding of OFDM signals affected by clipping. Multi-Carrier Spread Spectrum. 6, 443-452 (2006)

11. H Saeedi, M Sharif, F Marvasti, Clipping noise cancellation in OFDM systems using oversampled signal reconstruction. IEEE Commun Lett. 6(2), 73-75 (2002). doi:10.1109/4234.984699

12. S Wei, DL Goeckel, PA Kelly, A modern extreme value theory approach to calculating the distribution of the peak-to-average power ratio in OFDM systems, in IEEE ICC 2002 (2002)

13. S Wei, DL Goeckel, PA Kelly, Convergence of the complex envelope of bandlimited OFDM signals. IEEE Trans Inf Theory. 56(10), 4893-4904 (2010)

14. A Papoulis, SU Pillai, Probability, Random Variables and Stochastic Processes, 4th edn. (McGraw-Hill, 2002)

15. GA Korn, TM Korn, Mathematical Handbook for Scientists and Engineers (Dover, 2000)

doi:10.1186/1687-6180-2011-135

Cite this article as: Yoo et al:: Probability distribution analysis of MQAM-modulated OFDM symbol and reconstruction of distorted data. EURASIP Journal on Advances in Signal Processing 2011 2011:135.

\section{Submit your manuscript to a SpringerOpen ${ }^{\odot}$ journal and benefit from:}

- Convenient online submission

- Rigorous peer review

- Immediate publication on acceptance

- Open access: articles freely available online

- High visibility within the field

- Retaining the copyright to your article

Submit your next manuscript at $\boldsymbol{s p r i n g e r o p e n . c o m ~}$ 